\title{
Dysphagia in Patients with Neck Dissections
}

\author{
Zornitsa Mihaylova
}

Department of Dental, Oral and Maxillofacial Surgery, Faculty of Dental Medicine, Medical University Sofia, Bulgaria

\begin{abstract}
:
A common complication in patients with malignancies in the maxillofacial and cervical area is the impaired swallowing, due to the progress of the neoplasm or as a result of the treatment applied. Operative methods for the management of malignancies in the maxillofacial and cervical areas may damage anatomical structures, therefore causing swallowing disorders.

The purpose of the present study is to determine the degree of involvement of the swallow function depending on the volume of surgery - neck dissection.

Methods: SSQ (Sydney Swallowing Questionnaire) tool was used to assess the presence of dysphagia in operated patients, as well as an impaired swallowing function. The results obtained were analyzed by SPSS Vers23.0.

Results. Prospectively longitudinal study included 68 patients (25 women and 43 men) at mean age of 61.18 years (SD - 13.66). The reliability of the SSQ tool is assessed via Cronbach's coefficient alpha $\alpha=$ 0.871, and the validity of the sample is calculated using the Kaiser-Meyer-Olkin Measure of Sampling Adequacy (KMO coefficient) - 0.857. In the postoperative period, no significant difference in dysphagia was found in terms of the volume of surgery - neck dissection.

Conclusion. The type and the volume of neck dissection has no effect on the postoperative speech disorders and swallowing.
\end{abstract}

Key words: dysphagia, neck dissection, , postoperative consequenses, Sydney Swallowing Questionnaire

\section{Introduction.}

Patients with oral cancer usually have a swallowing disorder, which is a result of both the stage of the disease and the outcomes of the treatment performed [1].Dysphagia and aspiration are potentially serious consequences of the treatment of oral cancer and cervical metastases. [2].

Surgical methods for the treatment of malignancies in the maxillofacial and cervical areas are associated with damage of certain anatomical structures, followed by swallowing disorders [1].

The regional lymph nodes status is one of the main prognostic factors for the survival of patients with malignancies in the maxillofacial and cervical areas. Therefore, cervical lymph nodes disease treatment is one of the most discussed areas in maxillofacial oncology $[3,4,5]$.

Neck dissections are procedures for the staging and treatment of malignancies in the maxillofacial and cervical areas [6].They consist of the removal of lymph nodes from specific areas of the neck, as well as in the removal, at a defined volume of surgical intervention, of non-lymphatic anatomical structures, i.e. $\mathrm{m}$. sternocleidomasstoideus (MSCM), v. jugularis internal (VJI), n. accessorius (N.XI). Therefore, some postoperative consequences and complications may arise [7, 8, 9]. The evaluation and analysis of the undesirable treatment outcomes are defined mainly as complications and are directed to certain structures nerves [10, 11] and blood vessels [12, 13, 14].

Swallowing impairment (dysphagia). Hutchenson et al. $[15,16]$ found that the level of neck dissection (the volume of surgery) did not induce a chronic dysphagia occurrence. When analyzing the dysphagia profile, three phenomena are evaluated: aspiration, strictures, and nutrition via the naso-gastral tube [13]. The observed phenomena associated with impaired swallowing function in operative interventions can be divided into: 
- Retention - it represents a retention of food and saliva in the oral cavity, valekullae epigloticae and hypopharynx [17].It is caused by paresis or cicatricial changes in the pharyngeal muscle.

- Nasal penetration (regurgitation) - represents food regurgitation in the nasal cavity, caused by velopharyngeal insufficiency or pharyngeal stenosis [14].

- Laryngeal penetration - the entry of liquids and food into the space between plica ariepiglotica and plica vocalis [14].

Food aspiration/penetration is a phenomen dominantly associated with impaired nerve regulation of swallowing [18]. The strictures represent late deformities of the upper gastrointestinal tract (after 90 days) and are associated with morphological deformity of the mesopharynx and hypopharynx [19,20].

Methods for the study of swallowing disorders. There are two main groups of methods for swallowing investigation:

- Biomechanical methods - video fluoroscopic swallowing examination, fibrooptic endoscopic swallowing examination (FESE), ultrasound examination, nuclear scintigraphy.

- Bio-psycho-social methods - the development of this group of methods for assessing swallowing disorders and the consequences is due to the following reasons: 1) Changes in the biomechanics of swallowing have little relevance of influencing and achieving the main goals of the treatment of dysphagia, as safe, adequate, independent and satisfactory nutrition and hydration in particular [16]; in fact, changes in the biomechanics of swallowing are poorly related to these conditions. 2) The number of interventions referred to as treatment is increasing and the term "management" is used more commonly and replaces "therapy" and "rehabilitation" in the multidisciplinary approach in patients with dysphagia [16]. 3) There is a growing need for functional assessment of the consequences from a patient perspective [16]. 4) The current binary model for evaluating dysphagia management outcomes - what has been achieved and what needs to be done [21]. The purpose of the present study is to evaluate impaired swallowing function (dysphagia) in primary surgically treated patients with neck metastatic disease due to malignancies in the maxillofacial and cervical areas.

\section{Materials and methods.}

Patients included. A prospective study was performed including patients, treated in Maxillofacial surgery clinic, University Hospital-Alexandrovska with cervical metastasis arising from malignancies in the maxillofacial and cervical areas. When selecting the patients, those with preoperative radiation therapy were excluded. All patients completed informed consent to participate in this study.

Instrument. The PRO (Patient Reported Outcomes), evaluated by questionnaires are a reproductive, safe and cost-effective method for assessing the severity of dysphagia, allowing chronological monitoring of changes and outcome of treatment interventions. [22].Using the instruments, one aspect of dysphagia (symptoms), or multiple aspects (symptoms and quality of life) are evaluated individually [18].

SSQ (Sydney Swallowing Questionnaire) was used to evaluate the swallowing impairment level, resulting from the surgical management of malignancies in the maxillofacial and cervical areas. SSQ has been developed to evaluate neuromyogenic oral and/or oropharyngeal dysphagia. It is constructed from 17 questions to evaluate and quantify patient-reported swallowing difficulties [23]. A visual-analog scale (VAS), 100mm long, is used to indicate the degree of difficulty, with the patient 's " $\mathrm{X}$ " marking the degree of difficulty. The left end of the scale reflects no problems in swallowing, the middle indicates moderate problems, and the right end of the scale indicates severe difficulties.

No disability

Swallowing is significantly impaired

The value of each question is determined by measuring the distance from the left edge of the scale to the patient's marking area. The total scale is calculated by summing the values of the 16 questions with a visualanalogue register. To this value is added the value of question 12, multiplied by 20 - thus converting to the remaining values the 5-point evaluation of this question. Thus, the total value of the assessment is 1700 units [24]. The higher values are the more severe swallowing damage is observed.

The SSQ tool is available for completion by patients preoperatively and postoperatively at 3 months. 
Statistical analysis. SPSS Vers23.0 was used for the statistical processing and analysis of all data obtained. A level of significance that rejects the null hypothesis is assumed to be $\alpha=0.05$. Descriptive statistic was used to represent the measures of central tendency and the scattering of variables. Comparative deductive statistics, parametric statistical tests are applied: one-way ANOVA, Leven dispersion homogeneity test, stability test for equality of arithmetic means - Brown-Forsyte, Welch. To verify the reliability of the measures, Cronbach's alpha coefficient is calculated. A factor analysis was applied to verify the construct validity of the measurement, with an assessment of the adequacy measure of the sample - Kaiser - Mayer Olkin Measure of Sampling Adequacy and a Bartlett test for sphericity.

\section{Results.}

A total of 68 patients with neck dissections were examined. The cohort is represented by 25 women and 43 men, with a mean age (SD) of 61.18 years (13.66\%). Radical neck dissection (RND) was performed in 31 patients (46\%), supraomohyoid neck dissection (SOHND) was performed in 30 patients, and selective neck dissection (SND) was performed in 7 patients (10\%).

Reliability of the instruments

In order to investigate the reliability of the SSQ tool used to assess symptomatic functional swallowing problems in patients with malignancies in the maxillofacial region, a statistical test was conducted to measure Cronbach's coefficient alpha. The reliability of the tool is $\alpha=0.871$, which indicates that it is a reliable tool for evaluating functional problems (Table 1).

Table 1. Reliability of SSQ

\begin{tabular}{|c|r|r|}
\hline $\begin{array}{c}\text { Cronbach's } \\
\text { Alpha }\end{array}$ & $\begin{array}{c}\text { Cronbach's Alpha Based } \\
\text { on Standardized Items }\end{array}$ & Number of Items \\
\hline, 871 &, 875 & 17 \\
\hline
\end{tabular}

\section{Validity of instruments}

In order to investigate the validity of SSQ for the evaluation of symptomatic functional swallowing problems in patients with malignancies in the maxillofacial region, confirmatory factor analysis was performed, using the principal component method and orthogonal rotation by Varimax with Kaiser normalization. The results show high values of the Kaiser-Meyer-Olkin Measure of Sampling Adequacy KMO coefficient - 0.857, which exceeds the recommended value of 0.60 [25], with the Bartlett Test of Sphericity being statistically significant $(\mathrm{p}<0.000)[26]$. The confirmatory factor analysis shows that the scale is a valid tool for evaluating functional disorders (Table 2).

Table 2. Validity of SSQ

\begin{tabular}{|l|l|r|}
\hline Kaiser-Meyer-Olkin Measure of Sampling Adequacy. &, 857 \\
\hline Bartlett's Test of Sphericity & Approx. Chi-Square & 994,928 \\
\cline { 2 - 3 } & df & 136 \\
\cline { 2 - 3 } & Sig. &, 000 \\
\hline
\end{tabular}

\section{Assessment of swallow function in various neck dissections}

An impairment of symptomatic swallowing component was evaluated using the SSQ tool. The evaluation was performed preoperatively and postoperatively at the $3^{\text {rd }}$ month.

Low levels of the total scale score for the three variants of neck dissections were registered preoperatively $\mathrm{X}=86.44, \mathrm{SD}=90.57, \mathrm{CI} 95 \%=[64.52,108.36]$ (Table 3, Fig. 1). 
Table 3. Distribution of values from the swallow function evaluation by SSQ according to the volume of cervical dissection

\begin{tabular}{|c|c|c|c|c|c|c|c|c|c|}
\hline 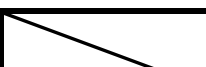 & Dysphagia & & & & & 95\% & CI & & \\
\hline $\begin{array}{l}\text { Type of } \\
\text { neck dissectio }\end{array}$ & on & $\mathbf{N}$ & $\overline{\mathrm{x}}$ & SD & SE & Lower & Upper & Min & Max \\
\hline SSQ & SOHND & 30 & 68,57 & 47,345 & 8,644 & 50,89 & 86,25 & 20 & $18 \varepsilon$ \\
\hline Preoperative & RND & 31 & 112,10 & 121,419 & 21,808 & 67,56 & 156,63 & 20 & 635 \\
\hline & SND & 7 & 49,43 & 21,306 & 8,053 & 29,72 & 69,13 & 26 & 67 \\
\hline & Total & 68 & 86,44 & 90,569 & 10,983 & 64,52 & 108,36 & 20 & 635 \\
\hline SSQ & SOHND & 30 & 851,33 & 431,470 & 78,775 & 690,22 & 1012,45 & 113 & 1574 \\
\hline Postoperative & RND & 31 & 1029,32 & 342,605 & 61,534 & 903,65 & 1154,99 & 113 & 1564 \\
\hline & SND & 7 & 735,43 & 420,588 & 158,967 & 346,45 & 1124,41 & 113 & 1245 \\
\hline & Total & 68 & 920,54 & 400,222 & 48,534 & 823,67 & 1017,42 & 113 & 157 \\
\hline
\end{tabular}

On the $30^{\text {th }}$ day postoperatively, significant swallowing function abnormalities were recorded: SOHND $\mathrm{X}=851.31, \mathrm{SD}=431.47, \mathrm{CI}_{95 \%}=[690.22,1012.45] ; \mathrm{RND}-\mathrm{X}=1029.32, \mathrm{SD}=342.61, \mathrm{CI}_{95 \%}=[903.65,1154.99]$; $\mathrm{SND}-\mathrm{X}=735.43, \mathrm{SD}=420.59, \mathrm{CI}_{95 \%}=[346.45,1124.41]$.

No statistically significant difference is established $(\mathrm{F}(2,65)=2.443, \mathrm{p}=0.095)$ in the swallow function, evaluated by the symptomatic SSQ scale, in neck dissections of different volumes.

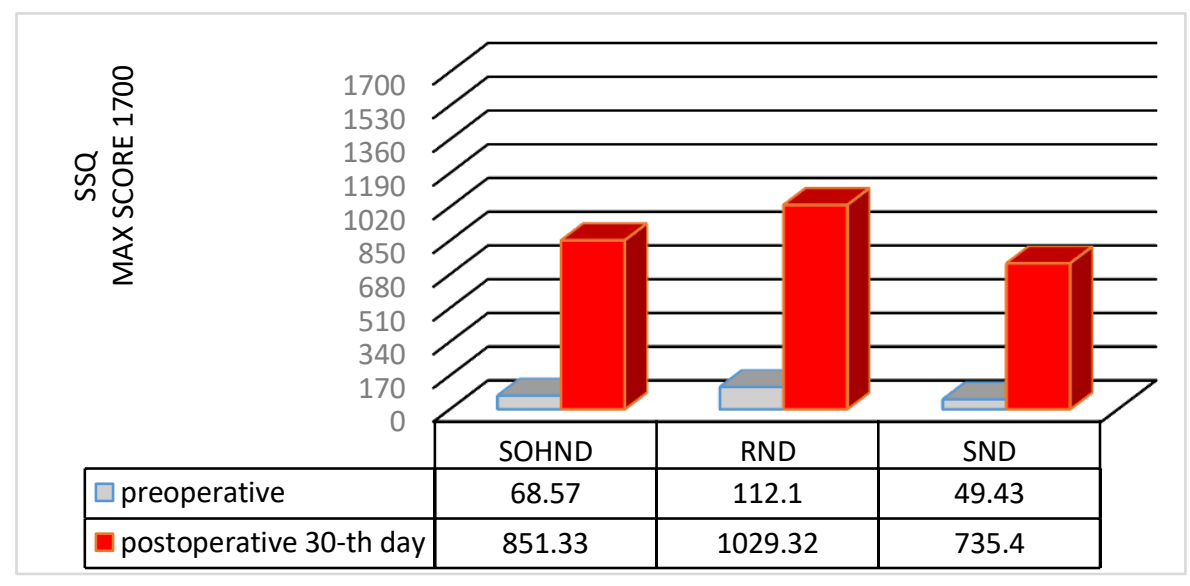

Figure 1. Swallowing function evaluation - SSQ, preoperatively and postoperatively in neck dissected patients

\section{Discussion}

Swallowing function. In the current study, no significant variability in the postoperative dysphagia was registered in the different types of neck dissection. Comparing the results of the symptomatic scale, it was found that the most obvious dysphagia is registered with RND, followed by SOHND. Hutchenson et al. [13] reveal that the extend of neck dissection (the volume of surgery) did not affect the development of chronic dysphagia. When analyzing the profile of dysphagia, three phenomena are evaluated: aspiration, strictures, and feeding by naso-gastral tube [13]. Food aspiration/penetration is a dominant phenomen associated with impaired nerve regulation of swallowing [18], while the strictures represent late deformities of the upper gastrointestinal tract (after 90 days) and are associated with morphological deformity of the mesopharynx and hypopharynx [18]. Due to the preservation of the neural structures and the reservation of the muscles of the meso- and hypopharynx, no differences in the degree of dysphagia were observed in the present study between the different groups of cervical dissections. [7].

\section{Conclusion.}

Dysphagia following surgical management of the metastases identified in the regional lymph nodes is most evident in RND, followed by SOHND and SND. Despite the descriptively observed differences in morbidity levels, no statistically significant difference in the present study was found between the three groups. 
[1] Kronenberger, M. B., A. D. Meyers. Dysphagia following head and neck cancer surgery. Dysphagia, 1994, 9, 236-244

[2] Shaker, Reza, Peter C. Belafsky, Gregory N. Postma, C. Easterling (eds). Principles of Deglutition. A Multidisciplinary Text for Swallowing and its Disorders. Springer, 2013, 1017 pp. ISBN 978-14614-3793-2, ISBN 978-1-4614-3794-9 (eBook)., DOI 10.1007/978-1-4614-3794-9

[3] Kirita, Tadaaki and Ken Omura (eds.). Oral Cancer: Diagnosis and Therapy. Springer Japan, 2015, 427 pp. ISBN 978-4-431-54938-3 (eBook), DOI 10.1007/978-4-431-54938-3

[4] Schuller, D. E., W. F. McGuirt, B. F. McCabe et al. The prognostic significance of metastatic cervical lymph nodes. Laryngoscope, 1980, 90, 557-570.

[5] Aleksiev, E. Functional consequences in surgical treatment in patients with maxillofacial and neck malignancy. - Medical Review, LIII, 2017, №4, 13-23

[6] Dedivitis, R. A., A. V. Guimaraes, E. G. Pfuetzenreiter Jr., M. A. F. de Castro. Neck dissection complications. Braz J Otorhinolaryngol., 2011, 77, 1, 65-69

[7] Aleksiev E, M Nikolova, M Kondeva, L Videnova, P Stanimirov. Comorbidity and functional consequences after operative treatment of malignancies in head and neck area: Part I: comorbidity and sensory function, pain, speech and swallowing. - Medical Review, LIII, 2017, №6, 41-46

[8] Aleksiev E, M Nikolova, M Kondeva, L Videnova, P Stanimirov. Comorbidity and functional consequences after operative treatment of malignancies in head and neck area Part II: comorbidity, musculo-skeletal function and mental status. - Medical Review, LIV, 2018, №1, 34 - 40

[9] Aleksiev, E. Assessment of functional consequences after operative treatment of malignancy in maxillofacial and neck area. Dissertation thesis, Sofia, 2018. (In Bulgarian)

[10] Aleksiev E., L. Videnova, M. Petkova, M. Zekov, P. Stanimirov. Pain and sensory dysfunction in neck dissection due to neck metastatic diseases in malignant tumors at maxillofacial region. Modern Dentistry, XLVIII, 2017, №1-2, 10-19

[11] Prim, M. P., J. I. de Diego, J. M. Verdaguer, N. Sastre, I. Rabanal. Neurological complications following functional neck dissection. Eur Arch Otorhinolaryngol., 2006, 263, 5, 473-476

[12] Tsekov M., M Mireva, K Guirov, S Marangozov, E. Aleksiev, Ch Videnova. Postsurgical rehabilitation of patients with superficial thrombophlebitis. Zdrave I nauka, VIII, 2018, 4, 35-38 (In Bulgaarian)

[13] Tsekov, M., K. Guirov, S. Marangozov, M. Mireva, E. Aleksiev, I. Lozev. Indications for emergency surgical intervention on superficial thrombophlebiti's patients. - Military medicine, 70, 2018, 2, 6265

[14] Tsekov, M., K. Guirov, S. Marangozov, M. Mireva, E. Aleksiev, I. Lozev. Superficial thrombophlebitis - a social aspect of the disease. - Military medicine, 70, 2018, 4, 41-43.

[15] Hutcheson, K. A., A. R. Abualsamh, A. Sosa, R. S. Weber, Beth M. Beadle, E. M. Sturgis, Jan S. Lewin. Impact of selective neck dissection on chronic dysphagia after chemo-intensity-modulated radiotherapy for oropharyngeal carcinoma. Head \& Neck, 2016, 886-892

[16] Aleksiev E, K.Guirov, B.Guirova, A.Mihova, E.Titianova. Clinical, Intraoperative and Multimodal Ultrasound Imaging Study of Facial Melanoma. - Neurosonology and Cerebral Hemodynamics, 10, 2014/1, 15-21

[17] Dysphagia. Diagnosis and Treatment. Olle Ekberg Editor. Medical Radiology. Diagnostic Imaging. Series Editors Maximilian F. Reiser Hedvig Hricak Michael Knauth. Springer-Verlag, 2012, 615 pp

[18] Miller, Arthur J. Overview of Deglutition and Digestion. In: Part I. General Aspects of Deglutition. Principles of Deglutition. A Multidisciplinary Text for Swallowing and its Disorders. Springer, 2013, 3-18

[19] Cichero, Julie A. Y., Bruce E. Murdoch. Dysphagia: Foundation, Theory and Practice. John Wiley, 2006, 582 pp. ISBN-10: 1861565054, ISBN:-13: 9781861565051

[20] Ilieva A., R. Kolarov, E. Aleksiev. The use of nutridrink in patients with surgical diseases in the maxillofacial region. - Medical Review, XLIX, 2013, №1, 50-55

[21] Khaldoun, E., V. Woisard, E. Verin. Validation in French of the SWAL-QOL scale in patients with oropharyngeal dysphagia. Validation de la version française du SWAL-QOL spécifique aux troubles de la déglutition. Gastroentérologie Clinique et Biologique, 2009, 33, 167-171 
[22] Miller, Arthur J. Overview of Deglutition and Digestion. In: Part I. General Aspects of Deglutition. Principles of Deglutition. A Multidisciplinary Text for Swallowing and its Disorders. Springer, 2013, 3-18

[23] Dwivedi, R. C., S. St. Rose, E. J. Chisholm, C. Georgalas, B. Bisase, F. Amen, C. J. Kerawala, P. M. Clarke, C. M. Nutting, P. H. Rhys-Evans, K. J. Harrington, R. Kazi. Evaluation of Swallowing by Sydney Swallow Questionnaire (SSQ) in Oral and Oropharyngeal Cancer Patients Treated with Primary Surgery. Dysphagia, 2012, 27, 4, 491-497

[24] Szczesniak, M. M., J. Maclean, T. Zhang, R. Liu, I. J. Cook. The Normative Range for and Age and Gender Effects on the Sydney Swallow Questionnaire (SSQ). Dysphagia, 2014, 29, 5, 535-538

[25] Kaiser, H. F. An index of factorial simplicity. Psychometrika, 1974, 39, 31-36

[26] Bartlett, M. S. A note on the multiplyung factors for various chi square approximations. J. Royal Statist. Soc., 16 (Series B), 1954, 296-298 